\title{
Cystatin C Deficiency Promotes Inflammation in Angiotensin II-Induced Abdominal Aortic Aneurisms in Atherosclerotic Mice
}

\author{
Stephanie Schulte, Jiusong Sun, Peter Libby, \\ Lindsey MacFarlane, Chongxiu Sun, \\ Marco Lopez-Ilasaca, Guo-Ping Shi, \\ and Galina K. Sukhova \\ From the Department of Medicine, Brigham and Women's \\ Hospital, and Harvard Medical School, Boston, Massachusetts
}

An imbalance between cysteinyl cathepsins and their principal endogenous inhibitor cystatin C (CystC) may favor proteolysis in the pathogenesis of human abdominal aortic aneurysms (AAA), yet a direct role of CystC in AAA remains unproven. This study used CystC and apolipoprotein E (ApoE) compound mutant $\left(\mathrm{CystC}^{-/-} \mathrm{ApoE}^{-/-}\right)$mice to examine directly the role of cysteine protease/protease inhibitor imbalance in AAA formation in angiotensin II-induced AAA. CystC-deficiency increased lumenal diameter and lesion size compared with control mice. $\mathrm{CystC}^{-/-}$ $\mathrm{ApoE}^{-/-}$lesions also demonstrated enhanced inflammatory cell accumulation, more severe elastin fragmentation, and fewer smooth muscle cells in the tunica media. Macrophage content, measured as percent positive area $(23.2 \pm 1.4 \%$ versus $11.2 \pm 1.4 \% ; P=0.0003)$ and number of the $\mathrm{CD}_{4}^{+} \mathrm{T}$ cells (ninefold; $P=0.048$ ), increased significantly in $\mathrm{CystC}^{-/-} \mathrm{ApoE}^{-/-}$lesions. CystC deficiency increased cathepsin activity (5.5 fold; $P=0.001)$ in AAA, yielding greater elastin degradation and proangiogenic laminin-5 $\gamma 2$ peptide production, which may account for increased microvascularization in $\mathrm{CystC}^{-/-} \mathrm{ApoE}^{-/-}$compared with $\mathrm{ApoE}^{-/-}$lesions. Increased leukocyte adhesion molecule VCAM-1 expression and leukocyte proliferation might also promote inflammation in CystC-deficient AAA. These data indicate that CystC contributes to experimental AAA pathogenesis and that enhanced cysteine protease activity, due to the lack of CystC, favors inflammation in AAA lesions induced in atherosclerotic mice by promoting microvascularization and smooth muscle cell apoptosis as well as leukocytes adhesion and proliferation. ( $\mathrm{AmJ}$ Pathol 2010, 177:456-463; DOI: 10.2353/ajpath.2010.090381)
The incidence of abdominal aortic aneurysm (AAA) continues to rise, necessitating 46,000 operations and causing about 15,000 deaths annually in the United States. ${ }^{1}$ The mostly descriptive knowledge of the pathoanatomy of $A A A,{ }^{2,3}$ the lack of biomarkers for aneurysm growth and rupture, and limited understanding of the mechanisms of AAA render surgical or percutaneous intervention the only effective treatment thus far.

Common morphological characteristics of AAA include chronic transmural inflammation characterized by abundant leukocyte infiltrates, degradation of medial elastin, depletion of medial smooth muscle cells (SMCs), and extensive neovascularization. ${ }^{4-8}$ Inflammatory cells including macrophages, lymphocytes, dendritic cells, neutrophils, and mast cells accumulate in AAA lesions in humans and mice and contribute prominently to AAA development. ${ }^{5,9}$ Positive correlation between inflammatory infiltrates and aneurysmal enlargement, ${ }^{4,9}$ as well as regression of established AAA by limiting proinflammatory signaling in mice, ${ }^{10}$ suggests an important role of inflammation in AAA pathogenesis.

Loss of elastin relates closely to aneurysm dilatation. ${ }^{11}$ Recent findings that stabilization of elastin delayed AAA development and attenuated aneurysmal expansion in rats support the importance of elastin integrity and point to possible therapeutic approaches for AAA treatment. ${ }^{12}$ Various proteases can degrade elastin, including matrix metalloproteinases and serine proteases. ${ }^{13-15}$ Data from our group suggest an important role of the cysteine protease cathepsins $S, K$, and $L$ in AAA pathogenesis. We have demonstrated overexpression of these elastolytic and collagenolytic enzymes in both human atherosclerotic and AAA lesions. ${ }^{16,17}$ Cystatin $\mathrm{C}$ (CystC), the dominant endogenous inhibitor of cysteine proteases (CPs), inhibits cysteinyl cathepsins competitively. ${ }^{18}$ Inflammation diminishes CystC expression and/or secretion; for

Supported by National Institutes of Health grants HL67249 (to G.K.S.), HL080472 (to P.L.), and HL60942/HL88547/HL81090 (to G.P.S.).

Accepted for publication March 9, 2010.

Address reprint requests to Galina K. Sukhova, Ph.D., Cardiovascular Medicine, Brigham and Women's Hospital, 77 Avenue Louis Pasteur, NRB-730J, Boston, MA 02115. E-mail: gsukhova@rics.bwh.harvard.edu. 
example, alveolar macrophages from people who smoke cigarettes, a prominent risk factor for AAA, release 10 to $50 \%$ less CystC in vitro than macrophages from nonsmokers. ${ }^{19}$ We demonstrated that aneurysmal lesions have substantially decreased CystC levels compared with nondiseased arteries. Therefore, altered counterbalance between cysteinyl cathepsins and CystC may promote proteolysis within the arterial wall. ${ }^{20,21}$ Consistent with our findings, evaluation of expression of different proteases from growing and ruptured human AAAs revealed a fivefold increase of cathepsins $K$ and $L$, and a 30-fold increase in cathepsin S proteins, but $80 \%$ lower CystC protein levels, compared with nondiseased aortas. ${ }^{22}$ Furthermore, low serum CystC levels in AAA patients and their inverse correlation with AAA progression and expansion highlight the importance of CPs and inhibitor balance for AAA pathogenesis. ${ }^{20,23,24}$

This study tested the hypothesis that cathepsin/cystatin imbalance regulates the formation of angiotensin II (Ang II)-induced AAA in mice. ${ }^{25}$ The results provide further support for dysregulated proteinase activity as a critical influence on arterial remodeling.

\section{Materials and Methods}

\section{Animal Protocol}

$\mathrm{ApoE}^{-/-}$mice (C57BL/6, the Jackson Laboratories, Bar Harbor, ME) were initially crossbred with the CystC ${ }^{-1-}$ mice $(\mathrm{C} 57 \mathrm{BL} / 6 / 129 \mathrm{~S})^{26}$ to generate $\mathrm{CystC}^{+/-} \mathrm{ApoE}^{+/-}$mice, which were used subsequently to generate three littermate-controlled experimental groups: $\mathrm{CystC}^{+/+} \mathrm{ApoE}^{-/-}$, CystC ${ }^{-/-} \mathrm{ApoE}^{-1-}$, and $\mathrm{CystC}^{-/-} \mathrm{ApoE}^{+/+}$mice. Sixmonth-old male mice underwent subcutaneous implantation of an osmotic minipump (ALZET Scientific Products, Cupertino, CA, Model 1002) filled either with saline or an Ang II solution (500 ng/min/kg; Sigma, St. Louis, MO). After 28 days on a chow diet, systolic blood pressure was measured, animals were sacrificed, plasma collected, aortic diameter measured, and aortic lesions harvested for lesion characterization. ${ }^{27,28}$ Apoptosis was evaluated in mice infused with Ang II (1000 ng/min/kg) for 5 days. All procedures were approved by the Animal Research Committee of Harvard Medical School.

\section{Lipid, Lipoproteins, and Blood Pressure}

Serum total cholesterol, lipoprotein cholesterol, and triglyceride concentrations were determined using enzymatic assay kits according to the manufacturer (Pointe Scientific, Inc., Canton MI).

We measured systolic blood pressure using a noninvasive tail cuff method and a PC-based data analyzer (BP 2000, Visitech Systems, Inc., Apex, NC). Three to four measurements were obtained from each nonanesthetized mouse (6 mice per group).

\section{Aneurysmal Lesions Characterization}

This study used a previously published approach to quantification of experimental aneurysms. ${ }^{27,28}$ We mea-
A

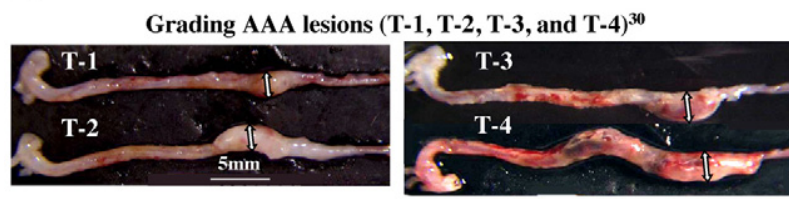

B

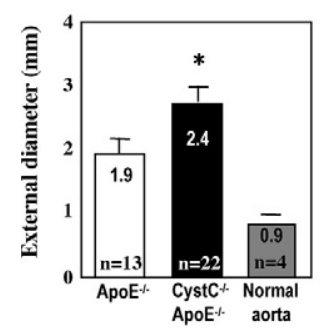

C

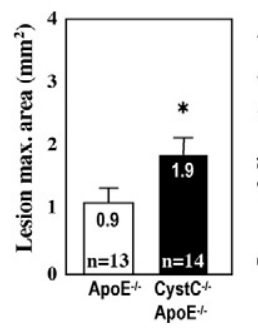

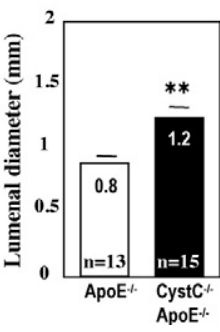

Figure 1. Cystatin $\mathrm{C}$ deficiency enhances aortic diameter and size of AAA lesions. A: Microphotographs show representative aortae graded by Daugherty et $\mathrm{al}^{27}$ (T-1 expanded aorta, T-2 small bulbous AAA lesions, T-3 bulbous T-2-like lesion with thrombus, and T-4 multiple AAA lesions with thrombus). Double arrows indicate maximal aortic diameter. Scale bar indicates magnification. B: Maximal aortic diameters were measured under physiological blood pressure in anesthetized mice. ${ }^{*} P<0.04$. C: Lesion area and lumenal diameter were measured on cross sections. Number of animals per group $(n)$ is indicated. ${ }^{*} P<0.03$, **: $P<0.02$

sured the percentage of incidence per group, evaluated lesion severity with grading keys, ${ }^{27}$ measured maximal external AAA diameter under physiological blood pressure while mice were anesthetized. We used OCT-embedded aneurysmal tissue for immunohistochemical analysis and snap-frozen AAA segments for protein extraction. Saraff et $\mathrm{al}^{28}$ previously described the complex pathology of Ang II-induced mouse AAA lesions. Intramural hematoma develops in the dissection area, inducing leukocyte infiltration in the hematoma and tunica media between 14 and 28 days. ${ }^{28}$ Because the influence of $\mathrm{CP}$ activity on the inflammatory response during AAA development was a key interest, we used 28 days of Ang II infusion for AAA analysis. All AAA tissue segments for immunohistochemistry were obtained from the maximal external aneurysm diameter (Figure 1A, double-headed arrows) $)^{27}$ at the level of medial elastica breaks. Ten slides per sample (three sections per slide) were numbered consecutively, and slides from identical levels were used for staining with each antibody.

\section{Immunohistochemical Analysis}

Serial cryostat cross sections $(6 \mu \mathrm{m})$ were used for immunostaining for macrophages (Mac-3, 1:900), T cells (CD4, 1:90), endothelial cells (ECs) (CD31, 1:1500) (BD Pharmingen, Franklin Lakes, NJ), SMCs ( $\alpha$-actin, FITCconjugated, 1:750, Sigma), apoptosis (TUNEL, Chemicon, Billerica, MA), proliferation (Ki-67, Novocastra Laboratories Ltd., Newcastle upon Tyne, UK), and laminin-5 $\gamma 2(1: 250) .{ }^{29}$ Data were analyzed as we described previously. ${ }^{9}$ We used incubation with appropriate IgG as a control of antibody specificity (not shown). The percentage of lesion area with positive color to total area for each section was recorded. Two observers blinded to the ori- 
gin of the samples analyzed all data. For double fluorescent immunohistochemistry, we mixed mouse cell typespecific antibodies conjugated with fluorochrome: CD11b-FITC or CD3-PE with rabbit anti-Ki-67 (cell proliferation). Staining for proliferation used biotinylated antirabbit secondary antibody followed by streptavidin conjugated with Texas Red (for costaining with CD11b) or with FITC (for costaining with CD3).

\section{Statistical Analysis}

Data were presented as mean \pm SE. Difference between groups was analyzed statistically by the nonparametric Mann-Whitney test. The degree of correlation between two variables was evaluated by linear regression analysis and by calculating the respective coefficient of determination $r^{2}$. $P$ values $<0.05$ were considered significant.

\section{Cysteine Protease Active Site Labeling}

To quantify cathepsin activity, we labeled aortic tissue extracts with biotinylated JPM, an active site-directed probe that irreversibly binds to active sites of cathepsins. ${ }^{9,16}$ Microdissected AAA tissues were pulverized and lysed in a pH 5.5 buffer. ${ }^{16}$ Protein extracts $(2 \mu \mathrm{g} /$ sample) were incubated with $1 \mu$ l of biotin-JPM (5 $\mu \mathrm{mol} / \mathrm{L}$ ) for 1 hour at $37^{\circ} \mathrm{C}$, followed by separation on a $14 \%$ SDS-PAGE for immunoblot analysis with horseradish peroxidase-conjugated avidin (1:10,000, Sigma) and visualization using the ECL-detection kit (Amersham Pharmacia, Buckinghamshire, UK). Individual cysteinyl cathepsins were identified by their relative molecular weights. Densitometric analysis measured the relative amount of total cathepsin activities of each sample. Data were presented as fold increase in cathepsin activity compared with the $\mathrm{ApoE}^{-1-}$ control aortae (mean $\pm \mathrm{SE}, n=3$ per group).

\section{Aortic Ring Assay}

An aortic ring assay was used to test CPs function in endothelial tubule formation as previously described. ${ }^{9}$

\section{In Situ Zymography}

We determined elastolytic activity in situ in $8-\mu \mathrm{M}$ unfixed cryostat sections from aneurysmal lesions using elastin conjugated with quenched fluorescein, which requires cleavage by elastolytic enzymes to become fluorescent, as a substrate (DQ-elastin; Molecular Probes, Eugene, OR). ${ }^{9}$ Elastolytic activities were determined in two separate experiments with conditions optimized for $\mathrm{CP}$ or matrix metalloproteinase (MMP). CP relative input was determined using an EDTA-containing $\mathrm{pH}-5.5$ buffer, with or without the nonselective cathepsin inhibitor E64d (20 $\mu \mathrm{mol} / \mathrm{L})$; MMP activity was determined in an E64d-containing pH-7.4 buffer, with or without the metalloenzyme inhibitor EDTA (20 mmol/L). Sections were examined under a fluorescent microscope with all images captured under the same settings and shutter conditions. The elas- tic laminae in the tunica media showed autofluorescence, this region was therefore excluded from analysis of elastolytic activity.

\section{SMC Isolation and in Vitro Apoptosis Assay}

SMCs were isolated from mouse aortas as described previously. ${ }^{30}$ We plated $1 \times 10^{4} \mathrm{SMC} / \mathrm{cm}^{2}$ onto an 8-chamber slide and incubated overnight with or without $0.06 \mathrm{mmol} / \mathrm{L}$ pyrrolidinedithiocarbamate (PDCT, Sigma). ${ }^{9}$ Staining for apoptosis (TUNEL) was performed according to the manufacturer's recommendations (Intergen Discovery Products). Slides were mounted with DAPI-containing media (Vectashield, Vector, Laboratories, Burlingame, CA).

\section{Results}

\section{CystC Deficiency Increases AAA Lesion Size and Diameter in $A p o E^{-/-}$Mice}

AAA incidence and severity evaluated 28 days after Ang II infusion ${ }^{27,28}$ did not differ significantly between groups. External AAA diameter, measured by a micrometer ocular at the areas of maximal aortic thickness under physiological blood pressure (Figure 1A, double-headed arrows), ${ }^{27}$ was significantly larger in mice deficient in CystC $(2.4 \pm 0.2$ versus $1.9 \pm 0.2 \mathrm{~mm}, P<0.04)$ compared with the control ApoE ${ }^{-1-}$ group (Figure 1B). Further evaluation of cryostat cross-sections, taken from aortic segments with maximal external AAA diameters, could yield smaller (shrunk) luminal diameters due to the manipulations required for frozen tissue processing. However, CystC-deficient mice had larger lesion size $(1.9 \pm 0.3$ versus $0.9 \pm 0.2$ $\left.\mathrm{mm}^{2}, P<0.03\right)$ and aortic lumen diameter $(1.2 \pm 0.09$ versus $0.8 \pm 0.03 \mathrm{~mm}, P<0.02)$ compared with the controls (Figure 1C). Ang II-infused CystC ${ }^{-/-} \mathrm{ApoE}^{+/+}$mice and saline-infused $\mathrm{CystC}^{-1-} \mathrm{ApoE}^{-1-}$ mice did not develop AAA (controls).

\section{CystC Deficiency Does Not Alter Systolic Blood Pressure, Low-Density Lipoprotein, or High-Density Lipoprotein Levels in Mice}

Ang II can affect systolic blood pressure. However, we did not detect any systolic blood pressure differences between the mice with and without CystC expression 28 days after Ang II infusion (122 \pm 9 in CystC $^{-1-}$ ApoE $^{-1-}$ versus $122 \pm$ $18 \mathrm{~mm} \mathrm{Hg}$ in control group, $P=0.8, n=6$ per group). Thus, increased AAA size in CystC ${ }^{-1-} \mathrm{ApoE}^{-1-}$ mice did not result from altered blood pressure.

Serum cholesterol levels correlate strongly with atherosclerosis. In mice with diet-induced atherosclerosis, absence of CystC increased serum lipid levels. ${ }^{31}$ However, CystC deficiency did not affect serum lipid levels among chow diet-fed Ang II-infused $A p o E^{-1-}$ mice (data not shown). These data correspond with the findings in humans that serum lipid levels do not correlate with AAA expansion. ${ }^{32}$ 
A $\mathrm{CystC}^{+/+} \mathrm{ApoE}^{-/}$

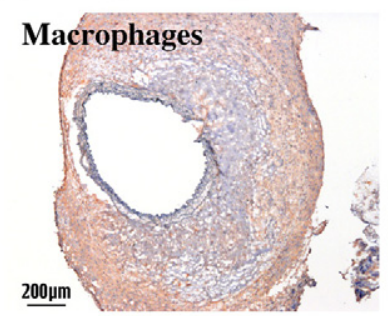

B

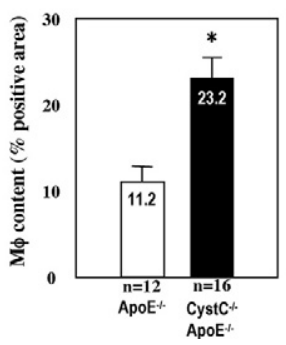

CystC $^{-/-A p p o E^{-/}}$

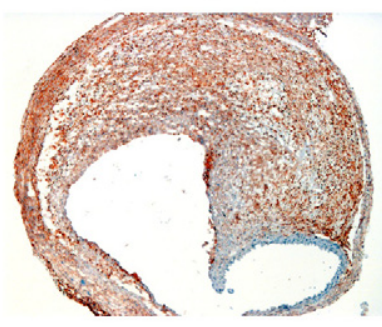

C

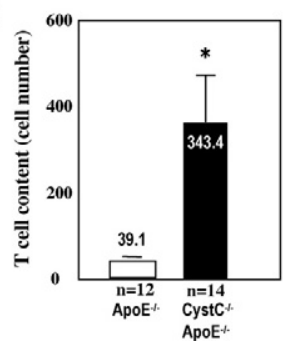

Figure 2. CystC deficiency increases inflammation in AAA lesions in mice A: Microphotographs of representative AAA lesions from $\mathrm{CystC}^{-/-} \mathrm{ApoE}^{-/-}$ and control mice stained with specific anti-murine macrophage antibody. B: Macrophage content measured as percentage of the entire lesion area stained for macrophages ${ }^{*} P<0.0003$. C: T cell content presented as number of CD4-positive cells per maximal cross section of AAA * $P<0.05$. Magnification indicated by scale bar.

\section{CystC Deficiency Increases Leukocyte Accumulation in AAA Lesions}

Leukocytes likely promote AAA formation by releasing cytokines, chemokines, proteases, and growth factors and mediating immune responses. Mice lacking these cells resist experimental AAA. ${ }^{9,33,34}$ Immunostaining revealed much larger macrophage-positive areas in AAA lesions from $\mathrm{CystC}^{-1-} \mathrm{ApoE}^{-1-}$ mice than in those from

CystC $^{+/+} \mathrm{ApoE}^{-/-}$mice $(23.2 \pm 1.4 \%$ versus $11.2 \pm$ $1.4 \% ; P<0.0003$ ) (Figure 2, A and B). CystC ${ }^{-l-} \mathrm{ApoE}^{-1-}$ lesions also had ninefold more $\mathrm{CD} 4^{+} \mathrm{T}$ cells compared with $\mathrm{CystC}^{+/+} \mathrm{ApoE}^{-/-}$mice $(343.4 \pm 121.7$ versus $39.1 \pm 7.2$ cells $/ \mathrm{mm}^{2} ; P<0.05$ ) (Figure $2 \mathrm{C}$ ). The majority of leukocytes localized in the periadventitial space but also in the hematoma and the tunica media, where leukocyte accumulation colocalized with elastica fragmentation and loss.

\section{AAA Lesions from CystC-Deficient Mice Demonstrate Enhanced Medial Elastica Degradation}

Within AAA lesions, leukocytes probably furnish most of the elastolytic cathepsins ${ }^{35}$ that may facilitate fragmentation of the medial elastic laminae, a hallmark of human and mouse AAA. We tested the hypothesis that increased accumulation of leukocytes in $\mathrm{CystC}^{-I-} \mathrm{ApoE}^{-1-}$ mice promotes elastin degradation and rupture. Elastin integrity was graded after Verhoeff-van Gieson staining as follows: grade 1, noticeable interruption in the medial elastic laminae without change of luminal shape; grade 2 , small disruption in elastic laminae with bulbous extension of the aortic lumen; grade 3, prolonged disruption in the elastic laminae; and grade 4, ruptured medial elastica with extensive increase of the aortic lumen (Figure 3A). In agreement with our hypothesis, AAA lesions from Cyst ${ }^{-1-} \mathrm{ApoE}^{-1-}$ mice had significantly greater elastica loss than those from $\mathrm{CystC}^{+/+} \mathrm{ApoE}^{-/-}$mice $(3.5 \pm 0.3$ versus $1.9 \pm 0.4 ; P<0.004$ ) (Figure 3B). Higher grades of elastica rupture in CystC-deficient AAA lesions corresponded to greater length of these breaks on longitudinal
A

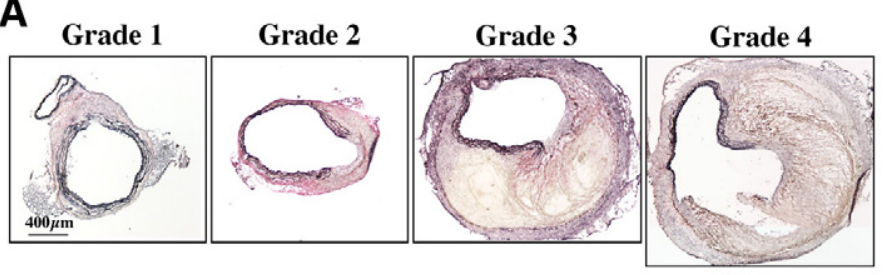

C
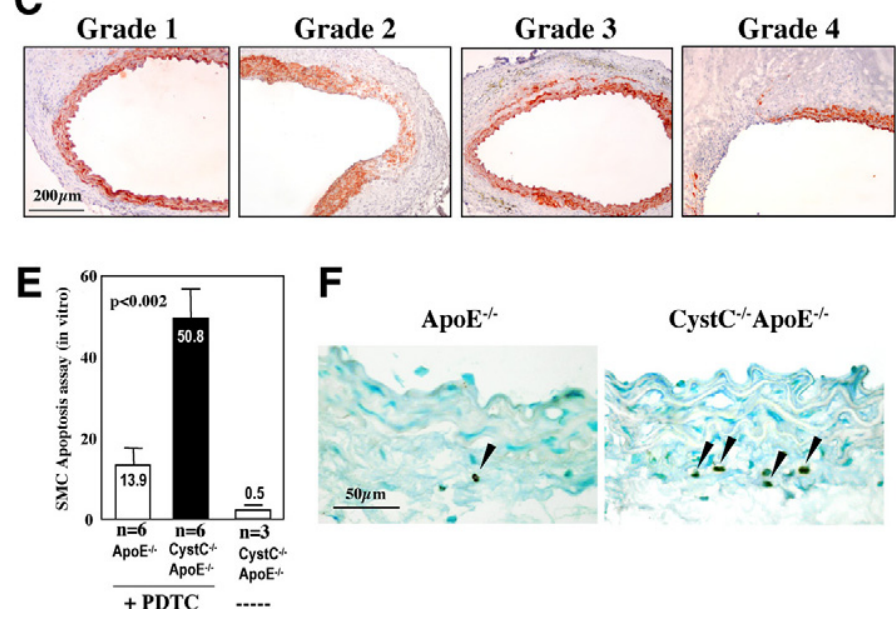

$\mathbf{F}$

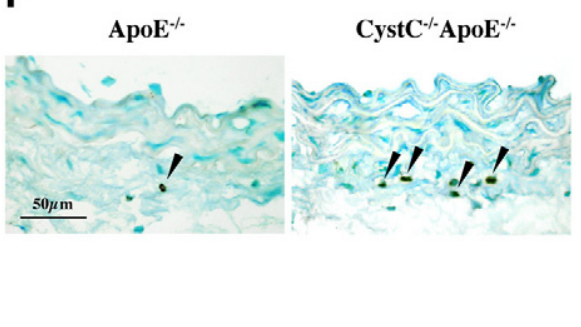

B

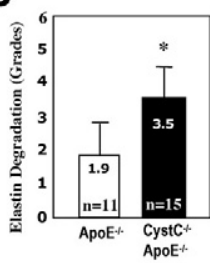

D

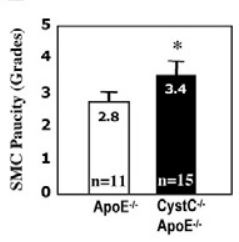

G

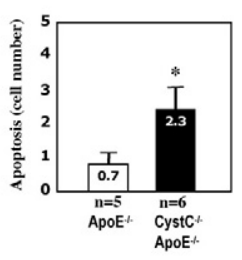

Figure 3. CystC deficiency increases elastica degradation and SMC loss in the tunica media in AAA lesions. A: Microphotographs represent grading scale for elastica (Verhoeff-van Gieson). B: Evaluation of elastica degradation by grading (higher scores indicate larger breaks/ruptures in elastica) ${ }^{*} P<0.004$. C: Microphotographs represent grading scale for SMCs (anti-mouse $\alpha$-actin). D: Evaluation of SMC preservation by grading (higher scores indicate more irregularity and loss of SMC) ${ }^{*} P<0.04$. E: Increased PDTCinduced apoptosis in SMCs isolated from CystC $^{-/-}$aortas compared with control SMCs Data present the percentage of apoptotic nuclei. F: Representative staining for apoptosis (TUNEL) in cross-sections from $\mathrm{ApoE}^{-/-}$(left) and $\mathrm{CystC}^{-/-} \mathrm{ApoE}^{-/-}$(right) suprarenal areas after five days of Ang II infusion. The arrowheads indicate apoptotic nuclei. G: Apoptotic SMC quantification. Data are presented as mean \pm SE. Number of animals indicated in each bar ${ }^{*} P<0.04$. 
sections $(299.3 \pm 21.3$ versus $93 \pm 16.5 \mu \mathrm{m} ; P<0.005$, $n=11$ per group).

\section{Paucity of SMCs in AAA Lesions from CystC-Deficient Mice}

Elastica degradation and disruption in the tunica media contribute directly to the loss of medial SMCs. Immunostaining for $\alpha$-actin revealed more pronounced disorganization and loss of medial SMCs in $\mathrm{CystC}^{-1-} \mathrm{ApoE}^{-/-}$ mice than in the control $\mathrm{ApoE}^{-1-}$ group. We graded SMC content in the tunica media using the following key: grade 1, intact SMCs; grade 2, minimal abnormalities, loss of few SMCs; grade 3, loss of SMC layers; and grade 4, loss of SMCs in prolonged areas of the tunica media (Figure 3C). ${ }^{9}$ Thus, the higher grades indicate poorer SMC preservation. Aortae of $\mathrm{CystC}^{-1-} \mathrm{ApoE}^{-1-}$ mice showed greater loss of SMCs than control mice (3.4 \pm 0.2 versus $2.8 \pm$ $0.2 ; P<0.04)$ (Figure 3D). SMCs appeared loosely arranged, irregularly distributed, and sparse in areas with disrupted elastin (Figure 3, A and C).

Apoptosis may also contribute to loss of SMCs in the tunica media. Indeed, SMCs isolated from the aortae of $\mathrm{CystC}^{-1-} \mathrm{ApoE}^{-1-}$ mice showed significantly greater susceptibility to PDTC-induced apoptosis than those from $\mathrm{CystC}^{+/+} \mathrm{ApoE}^{-/-}$aortas $(P<0.002$, TUNEL) (Figure $3 \mathrm{E})$. Although we observed SMC disorganization and loss in CystC-deficient mice, the total percentage of lesional apoptotic cells at 28-day time point did not differ between groups (not shown). At earlier stages of AAA formation we found significantly more apoptotic cells in CystC ${ }^{-1-} \mathrm{ApoE}^{-1-}$ aortic specimens (1000 ng/min $/ \mathrm{kg}$ Ang-II $1000 \mathrm{ng} / \mathrm{min} / \mathrm{kg}$ for 5 days) by analysis of TUNEL staining on cross-sections from five levels of the suprarenal aorta (60 $\mu \mathrm{m}$ apart) for each animal (Figure 3, F and G). In $\mathrm{CystC}^{-1-} \mathrm{ApoE}^{-1-}$ mice, increased elastica fragmentation and apoptosis of SMC may contribute to enhanced AAA diameter.

\section{CystC Deficiency Increases Elastolytic Activity in AAA Lesions}

Increased arterial elastin fragmentation in $\mathrm{CystC}^{-1-} \mathrm{ApoE}^{-1-}$ mice (Figure 3, A and B) suggests elevated elastolytic activities in their lesions compared with those in the control animals, presumably due to the lack of CystC and consequent increase of elastolytic cathepsin activities. Testing this hypothesis used active site labeling of cathepsins with biotinylated-JPM followed by quantification with a densitometric analysis using aneurysmal tissue extracts from both $\mathrm{CystC}^{+/+} \mathrm{ApoE}^{-1-}$ and $\mathrm{CystC}^{-/-} \mathrm{ApoE}^{-/-}$mice. Indeed, lesional extracts from $\mathrm{CystC}^{-1-} \mathrm{ApoE}^{-1-}$ mice showed a 5.5fold increase of total cathepsin activity over those from CystC $^{+/+} \mathrm{ApoE}^{-1-}$ controls (Figure 4A). We affirmed our conclusion in situ using a fluorogenic elastin substrate, which releases fluorescence on degradation, and unfixed frozen sections from AAA lesions. Consistent with our hypothesis, AAA from $\mathrm{CystC}^{-1-} \mathrm{ApoE}^{-1-}$ mice expressed higher elastolytic cathepsin activities (Figure 4B, bright
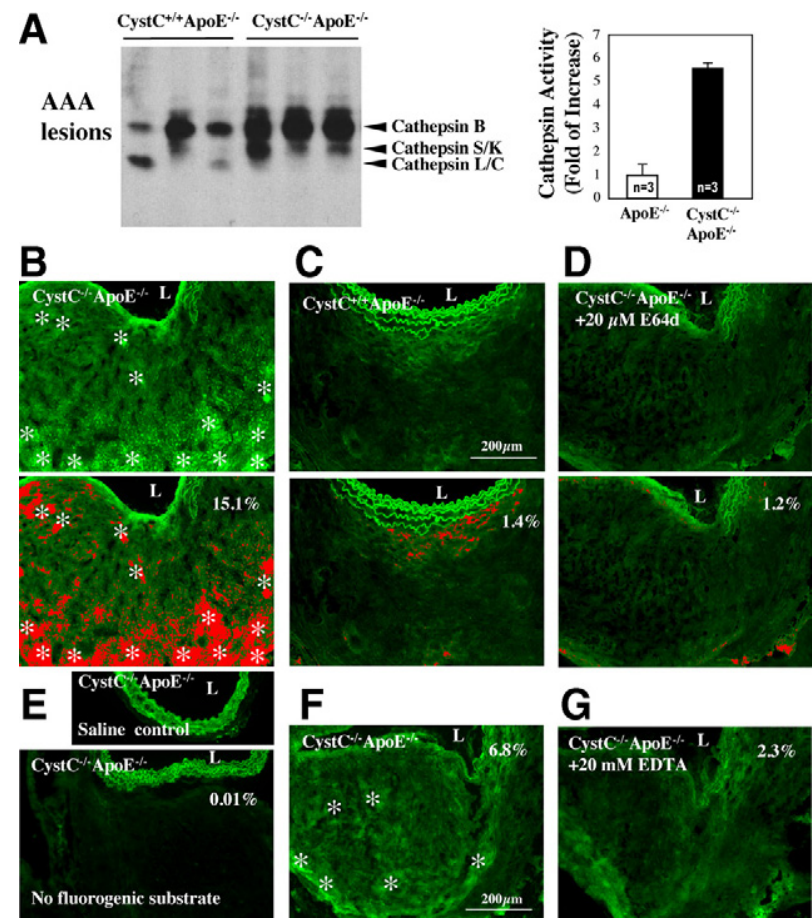

Figure 4. Increased elastolytic activity in AAA lesions from $\mathrm{CystC}^{-/-} \mathrm{ApoE}^{-/-}$ mice. A: Biotin-JPM cysteine protease active site labeling of AAA tissue extracts from $\mathrm{CystC}^{-/-} \mathrm{ApoE}^{-/-}$and $\mathrm{ApoE}^{-/-}$mice. Arrowhead indicates each active cathepsin. Densitometric analysis of cathepsin activity is shown in the right panel. Data are presented as mean $\pm \mathrm{SE}, P<0.05$ was considered significant ( $n=3) P<0.001$. B-G: In situ elastin zymography. B-D, top panels: Experimental conditions were optimized for cathepsin activity: pH 5.5 and 20 mmol/L EDTA. B: Asterisks indicate elastolytic cathepsin activity (green fluorescence) in CystC-deficient AAA lesions. C: Reduced elastolytic activity in control $\mathrm{CystC}^{+/+} \mathrm{ApoE}^{-/-}$aorta. D: The nonselective cathepsin inhibitor E64d $\left(20 \mu \mathrm{mol} / \mathrm{L}\right.$ ) abolished elastolytic activity in an adjacent section of $\mathrm{CystC}^{-}$ $-\mathrm{ApoE}^{-/-}$lesion. B-D, bottom panels: Elastolytic activity-dependent fluorescence is shown in red, and autofluorescence excluded from the analysis in green. The percentage of fluorescence intensity is indicated in each panel. $\mathbf{E}$ : Saline-treated aorta, and $\mathrm{CystC}^{-/-} \mathrm{ApoE}^{-/-}$aorta without DQ elastin substrate, were used as negative controls. F: Conditions were optimized for MMP activity: $\mathrm{pH}$ 7.4 and $20-\mu \mathrm{M}$ E64d. Asterisks indicate elastolytic MMP activity. G: Reduced elastolytic activity in an adjacent section treated with $20 \mathrm{mmol} / \mathrm{L}$ EDTA. Images represent three independent experiments. L indicates aortic lumen, scale bar $=200 \mu \mathrm{m}$

green fluorescence labeled by asterisks) than those from control mice (Figure 4 C). Because green fluorescence in the tunica media may result from increased elastase activity in lesions or from autofluorescence of the intact elastin filaments, which also appeared in saline-treated $\mathrm{CystC}^{-/-}$ $\mathrm{ApoE}^{-1-}$ aortas and in the absence of fluorogenic substrate (Figure 4E), we excluded this area from our analysis. We identified elastolytic activity-dependent fluorescence in red, leaving green autofluorescence of the tunica media nonselected (Figure 4, B-D, bottom panels). The cathepsin inhibitor E64d $(20 \mu \mathrm{mol} / \mathrm{L})$ diminished this activity in the parallel sections (Figure 4, B and D), as indicated by the differences in percentage area of lesional fluorescence (15.1 versus 1.2\%), while autofluorescence was unchanged (Figure 4, B and D, bottom panels). These data support a major contribution of CPs to elastolysis in this experimental AAA. Lesions from $\mathrm{CystC}^{-1-} \mathrm{ApoE}^{-1-}$ mice showed much less elastolytic MMP activity (compare Figure 4, B and F), and the metalloenzyme inhibitor EDTA (20 mmol/L) decreased this activity (Figure 4G). 
A
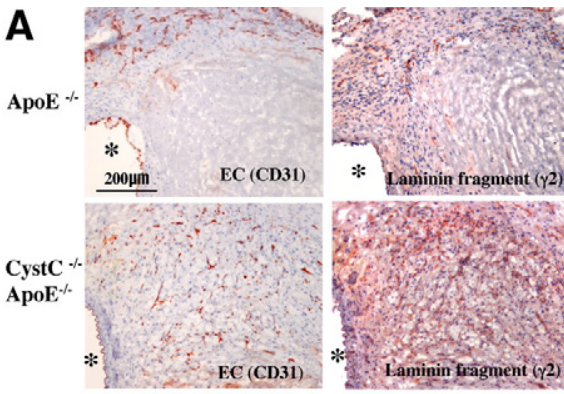

D

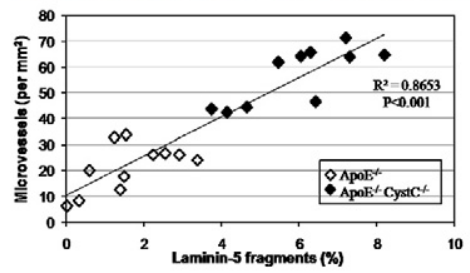

E

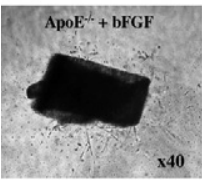

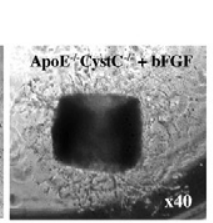

$\mathbf{F}$

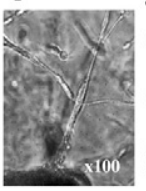

B

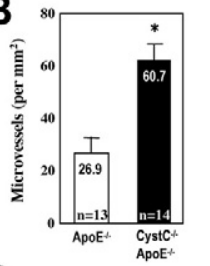

C

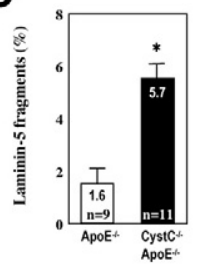

A

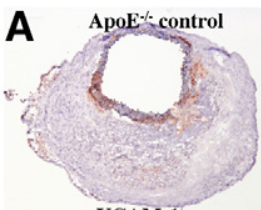

VCAM-1

$\mathrm{E}$

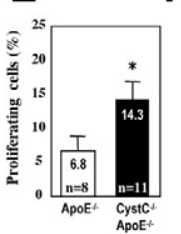

$\mathbf{F}$

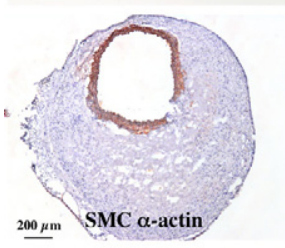

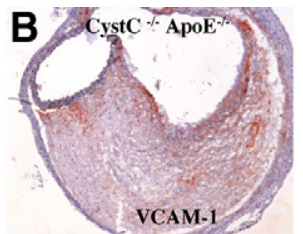

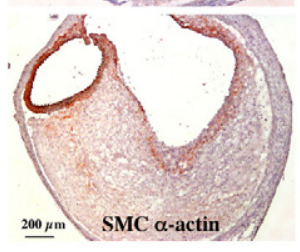

C

Non-AAA aorta

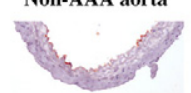

$\underline{200 \mu \mathrm{m}}$ VCAM-1
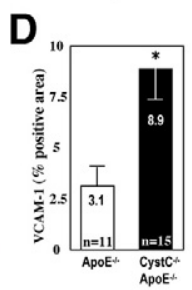

G

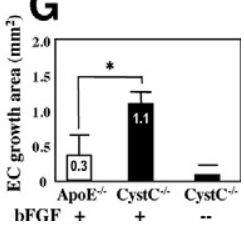

Figure 5. CystC-deficiency promotes microvascularization in AAA lesions. A: Immunostaining for ECs (CD31) and laminin- 5 fragment $\gamma 2$ on representative serial sections from $\mathrm{CystC}^{-1-} \mathrm{ApoE}^{-1-}$ lesion. Note colocalization of microvessels (left) with laminin-5 $\gamma 2$ fragments implicated in angiogenesis (right). Scale bar $=200 \mu \mathrm{m}$. Asterisks indicate aortic lumen. B: Number of CD31-positive microvessels $/ \mathrm{mm}^{2}$ was counted in cross-sections ${ }^{*} P<0.0004$. C: Laminin- $5 \gamma 2$ fragments measured as percentage of positive (red staining) area to the entire lesion area ${ }^{*} P<0.001$. D: Linear regression analysis of scatterplot of microvessel density versus laminin- $5 \gamma 2$ fragments content. Coefficient of determination $r^{2}=0.8653(n=21, P<0.001)$. E: Representative aortic rings from $\mathrm{ApoE}^{-/-}$and $\mathrm{CystC}^{-1} \mathrm{ApoE}^{-/}$mice immersed in Matrigel and stimulated with bFGF. F: Tubular structure of sprouting ECs on a high-magnification image. G: Statistical analysis of EC growth area ( $n=6$ per genotype). Media without bFGF was used as negative control. ${ }^{*} P<0.03$.

\section{Deficiency of CystC Increases}

Microvascularization, Adhesion Molecule

Expression, and Leukocyte Proliferation

\section{in AAA Lesions}

Microvessel growth may promote AAA formation, while inhibition of angiogenesis blocks AAA formation in mice. ${ }^{36}$ We hypothesized that increased inflammatory cell infiltration in $\mathrm{CystC}^{-1-} \mathrm{ApoE}^{-1-}$ mice relates to enhanced microvessel formation. Indeed, the total $\mathrm{CD} 31^{+}$microvessel numbers/ $\mathrm{mm}^{2}$ in AAA lesions from CystC ${ }^{-1-} \mathrm{ApoE}^{-/-}$mice increased significantly over those from $\mathrm{CystC}^{+/+} \mathrm{ApoE}^{-1-}$ mice $(60.7 \pm 4.6$ versus $26.9 \pm 3.5 ; P<0.0004)$ (Figure 5 , $A$ and $B$ ). We demonstrated previously that arterial neovascularization requires cathepsin $\mathrm{S},{ }^{37}$ which produces proangiogenic fragments $100 \mathrm{kDa} \gamma 2^{\prime}, 80 \mathrm{kDa} \gamma 2 \chi$, and $50 \mathrm{kDa}$ $\gamma 2^{\prime}$ peptides from laminin-5 proteolysis. ${ }^{29}$ Increased activity of CatS, a major target of CystC, ${ }^{35}$ in $\mathrm{CystC}^{-1-} \mathrm{ApoE}^{-1-}$ lesions (Figure 4, A and B) may contribute to enhanced microvascularization in AAA lesions of these mice (Figure $5 \mathrm{~A}$, left and $5 \mathrm{~B}$ ). Indeed, we detected abundant proangiogenic laminin-5 $\gamma 2$ fragments in $\mathrm{CystC}^{-1-} \mathrm{ApoE}^{-1-}$ lesions, 3.7-fold increase compared with control (Figure 5A, right and 5 C). Interestingly, laminin-5 $\gamma 2$ fragments colocalize
Figure 6. CystC-deficiency promotes inflammatory cells recruitment and proliferation in AAA lesions. Microphotographs of representative serial sections from $\mathrm{ApoE}^{-/-}$(A) and $\mathrm{CystC}^{-/-} \mathrm{ApoE}^{-/-}$(B) lesions stained for VCAM-1 (top) and SMC $\alpha$-actin (bottom). Note colocalization of VCAM-1 with SMCs. C: Nondiseased aorta showed VCAM-1 staining in luminal ECs only. D: Increased expression of VCAM-1 in $\mathrm{CystC}^{-/-} \mathrm{ApoE}^{-1-}$ lesions measured as percentage of positive (red stained) area to the entire lesion area. Scale bar $=200 \mu \mathrm{m}{ }^{*} P<0.002$. E: Percentage of Ki-67-positive proliferating cells was calculated for each AAA lesion ${ }^{*} P<0.05$. F: Colocalization of Ki-67-positive nuclei with $\mathrm{CD}_{11} \mathrm{~b}^{+}$macrophages (left) or $\mathrm{CD}^{+}{ }^{+} \mathrm{T}$ cells (right). Representative sections from $\mathrm{CystC}^{-/-} \mathrm{ApoE}^{-/-}$lesions demonstrate double-immunofluorescent staining (white arrowheads)

with microvessels detected by immunostaining on serial sections (Figure 5A). Furthermore, analysis of correlation between microvessel density (number of microvessels $/ \mathrm{mm}^{2}$ of lesion area) with laminin-5 fragments (percentage of lesion area stained for these peptides) showed a high degree of association $\left(r^{2}=0.8653, P<0.001\right)$ (Figure 5D). Our results suggest that these proangiogenic peptides further facilitate microvessel development and growth, promoting inflammatory cell infiltration during AAA formation in CystCdeficient mice. We also demonstrated threefold higher endothelial sprouting from the aortic rings of $\mathrm{CystC}^{-1-} \mathrm{ApoE}^{-1-}$ versus control $\mathrm{ApoE}^{-1-}$ mice, supporting a role for CPs in angiogenesis (Figure 5, E-G) during AAA development.

Increased inflammation in AAA lesions from $\mathrm{CystC}^{-/-}$ $\mathrm{ApoE}^{-/-}$mice (Figure 2) might result from enhanced recruitment of blood-borne leukocytes. To test this hypothesis, we measured VCAM-1 immunostaining in AAA lesions. In control ApoE ${ }^{-1-}$ lesions, VCAM-1 staining colocalized with SMCs within the tunica media and was higher compared with exclusively luminal EC staining in non-diseased aorta. As anticipated, AAA lesions from $\mathrm{CystC}^{-1-} \mathrm{ApoE}^{-1-}$ mice showed higher VCAM-1 expression (threefold, $P<$ 0.001 ) by SMCs in the tunica media and in the deeper lesion area (Figure 6, A-D). Similar to VCAM-1 distribution observed in atherosclerotic rabbits, its expression by ECs ceases after a few weeks, but SMCs begin to express this adhesion molecule. ${ }^{38}$ VCAM-1 produced by SMCs might promote the recruitment and retention of mononuclear cells in the arterial wall.

In addition AAA lesions from $\mathrm{CystC}^{-/-} \mathrm{ApoE}^{-/-}$mice had significantly elevated cell proliferation $\left(\mathrm{Ki}-67^{+}\right) \mathrm{com}$ - 
pared with those from CystC ${ }^{+/+} \mathrm{ApoE}^{-/-}$mice (twofold, $P<0.05)$ (Figure 6E). Most proliferating leukocytes localized in the periadventitial space, but some populated the hematoma and the tunica media. Double-immunofluorescent labeling revealed proliferation of both CD3-positive $T$ cells and CD11b-positive macrophages in AAA lesions (Figure 6F), suggesting local proliferation of peripheral leukocytes in AAA.

\section{Discussion}

Increased cysteinyl cathepsin activities in human aneurysmal lesions suggest participation of this family of proteases in AAA pathogenesis. The present study tested whether a skewed balance of CPs and their endogenous inhibitor CystC promotes this arterial disease. Although no experimental preparation with AAA recapitulates precisely the human condition, current mouse models permit testing of mechanistic hypotheses regarding common elements of pathogenesis. We used Ang II-treated mice, as described by Daugherty and his colleagues, because this preparation encompasses some important features of human AAA, including systemic increase of angiotensin II in the peripheral blood, increased aorta diameter, degradation of elastic laminae in the tunica media, and inflammation. ${ }^{25,27,28}$ This study provides experimental evidence that supports the hypothesis that reduced CystC and increased cathepsin activities in human AAA do not occur merely as a result of AAA formation, but rather directly contribute to the pathogenesis.

Results from this study suggest that CystC plays at least four roles in AAA pathogenesis by regulating cathepsin activities. First, increased CP activity (Figure 4A) leads to enhanced transmural disruption of medial elastic laminae (Figure 3, A and B). These results agree with our earlier findings of increased medial elastica loss in atherosclerotic lesions of $\mathrm{CystC}^{-1-} \mathrm{ApoE}^{-1-}$ mice. ${ }^{31} \mathrm{We}$ have hypothesized previously ${ }^{31}$ that cellular mediators of inflammation, such as T lymphocytes, neutrophils, mast cells, and macrophages recruited from the lumen, regulate expression of CystC and cathepsins. Indeed, mice lacking $T$ cells, ${ }^{33}$ neutrophils, ${ }^{34}$ and mast cells ${ }^{9}$ resist experimental AAA formation. Thus, inflammatory cell recruitment probably contributes to the increase of cathepsins and decrease of CystC during AAA formation. ${ }^{20,39}$

Second, lack of CystC affects medial SMC apoptosis, an important feature of human AAA. In a recent study, induction of medial SMC proliferation by bFGF successfully limited aneurysm enlargement, highlighting the importance of SMC integrity in resisting AAA progression. ${ }^{40}$ Studies using genetically altered mice demonstrated that CatL and CatS could trigger cell apoptosis by direct activation of caspases $^{41,42}$ or indirectly by activation of the proapoptotic mediator Bid. ${ }^{43}$ Therefore, increased activity of cathepsins due to CystC deficiency may contribute to enhanced medial SMC apoptosis. Indeed, we observed more than threefold increased apoptosis (TUNEL) in vitro (PDTC-induced SMCs isolated from CystC-deficient compared with control aortas) (Figure $3 \mathrm{E}$ ) and in vivo (5 days of Ang II infusion) (Figure 3, F and G). Consequent SMC loss could contribute to tunica media weakening, and thereby favor AAA formation in these mice.
Third, extensive microvessel formation, an ongoing process that characterizes human AAA, occurs during the initial stage and in mature aortic aneurysms. ${ }^{8,44}$ The role of CPs in AAA pathophysiology extends beyond degradation of structural extracellular matrix; these enzymes also contribute to a number of processes through other substrates such as cytokines, integrins, and other proteases. ${ }^{45-47}$ We recently suggested a mechanism of microvessel growth stimulation by CatS by generating highly proangiogenic laminin- 5 $\gamma 2$ fragments. ${ }^{29}$ Consistent with this finding, we detected increased levels of such laminin-5 $\gamma 2$ fragments, colocalization of these proteins with extensive microvascularization, and strong correlation between these two variables $\left(r^{2}=0.8653, P<\right.$ 0.001 ) in AAA lesions of $\mathrm{CystC}^{-1-} \mathrm{ApoE}^{-1-}$ mice (Figure 5, A-D), supporting a role for CPs in angiogenesis.

Fourth, CystC deficiency accelerates cell proliferation in AAA lesions. Both T cells and macrophages proliferate in the areas of inflammatory cell accumulation (Figure 6F) and may thus contribute to the enhanced inflammatory phenotype detected in $\mathrm{CystC}^{-1-} \mathrm{ApoE}^{-1-}$ mice. CPs can target several molecules on human immune cells including the member of the $\beta_{2}$ integrin family, lymphocyte function-associated antigen-1 (LFA-1). ${ }^{46,48}$ LFA-1 induces $\mathrm{T}$-cell proliferation providing mechanistic explanation for the effect of CPs on leukocyte proliferation. ${ }^{49}$

In conclusion, this study demonstrates that the enhanced CPs activity in CystC-deficient mice promotes SMC apoptosis, lesion microvascularization, and leukocyte recruitment, proliferation, and accumulation in AAA lesions. These findings provide new insight into mechanisms by which dysregulation of proteases participate in AAA pathogenesis by targeting/activating molecules involved in the key cell functions. Degradomics, which offers a new opportunity to identify protease substrates in biological samples, will aid the discovery of new functions performed by CPs in vascular biology..$^{50}$

\section{Acknowledgments}

We thank Eugenia Shvartz and Juan Du for skillful technical support and Joan Perry for editorial assistance.

\section{References}

1. Stanley JC, Barnes RW, Ernst CB, Hertzer NR, Mannick JA, Moore WS: Vascular surgery in the United States: workforce issues. Report of the Society for Vascular Surgery and the International Society for Cardiovascular Surgery, North American Chapter, Committee on Workforce Issues. J Vasc Surg 1996, 23:172-181

2. Patel MI, Hardman DT, Fisher CM, Appleberg M: Current views on the pathogenesis of abdominal aortic aneurysms. J Am Coll Surg 1995, 181:371-382

3. Powell J, Greenhalgh RM: Cellular, enzymatic, and genetic factors in the pathogenesis of abdominal aortic aneurysms. J Vasc Surg 1989 9:297-304

4. Anidjar S, Dobrin PB, Eichorst M, Graham GP, Chejfec G: Correlation of inflammatory infiltrate with the enlargement of experimental aortic aneurysms. J Vasc Surg 1992, 16:139-147

5. Shimizu K, Mitchell RN, Libby P: Inflammation and cellular immune responses in abdominal aortic aneurysms. Arterioscler Thromb Vasc Biol 2006, 26:987-994 
6. Thompson RW, Liao S, Curci JA: Vascular smooth muscle cell apoptosis in abdominal aortic aneurysms. Coron Artery Dis 1997, 8:623-631

7. Henderson EL, Geng YJ, Sukhova GK, Whittemore AD, Knox J, Libby $P$ : Death of smooth muscle cells and expression of mediators of apoptosis by T lymphocytes in human abdominal aortic aneurysms. Circulation 1999, 99:96-104

8. Holmes DR, Liao S, Parks WC, Thompson RW: Medial neovascularization in abdominal aortic aneurysms: a histopathologic marker of aneurysmal degeneration with pathophysiologic implications. J Vasc Surg 1995, 21:761-771; discussion 771-762

9. Sun J, Sukhova GK, Yang M, Wolters PJ, MacFarlane LA, Libby P, Sun C, Zhang Y, Liu J, Ennis TL, Knispel R, Xiong W, Thompson RW, Baxter BT, Shi GP: Mast cells modulate the pathogenesis of elastaseinduced abdominal aortic aneurysms in mice. J Clin Invest 2007, 117:3359-3368

10. Aoki H, Yoshimura K, Matsuzaki M: Turning back the clock: regression of abdominal aortic aneurysms via pharmacotherapy. J Mol Med 2007, 85:1077-1088

11. Dobrin PB, Baker WH, Gley WC: Elastolytic and collagenolytic studies of arteries. Implications for the mechanical properties of aneurysms. Arch Surg 1984, 119:405-409

12. Isenburg JC, Simionescu DT, Starcher BC, Vyavahare NR: Elastin stabilization for treatment of abdominal aortic aneurysms. Circulation 2007, 115:1729-1737

13. Shapiro SD, Campbell EJ, Welgus HG, Senior RM: Elastin degradation by mononuclear phagocytes. Ann NY Acad Sci 1991, 624:69-80

14. Thompson RW, Parks WC: Role of matrix metalloproteinases in abdominal aortic aneurysms. Ann NY Acad Sci 1996, 800:157-174

15. Knox JB, Sukhova GK, Whittemore AD, Libby P: Evidence for altered balance between matrix metalloproteinases and their inhibitors in human aortic diseases. Circulation 1997, 95:205-212

16. Sukhova GK, Shi GP, Simon DI, Chapman HA, Libby P: Expression of the elastolytic cathepsins $\mathrm{S}$ and $\mathrm{K}$ in human atheroma and regulation of their production in smooth muscle cells. J Clin Invest 1998, 102:576-583

17. Liu J, Sukhova GK, Yang JT, Sun J, Ma L, Ren A, Xu WH, Fu H, Dolganov GM, Hu C, Libby P, Shi GP: Cathepsin L expression and regulation in human abdominal aortic aneurysm, atherosclerosis, and vascular cells. Atherosclerosis 2006, 184:302-311

18. Chapman HA Jr, Reilly JJ Jr, Yee R, Grubb A: Identification of cystatin C, a cysteine proteinase inhibitor, as a major secretory product of human alveolar macrophages in vitro. Am Rev Respir Dis 1990, 141:698-705

19. Rodriguez RJ, White RR, Senior RM, Levine EA: Elastase release from human alveolar macrophages: comparison between smokers and nonsmokers. Science 1977, 198:313-314

20. Shi GP, Sukhova GK, Grubb A, Ducharme A, Rhode LH, Lee RT, Ridker PM, Libby P, Chapman HA: Cystatin C deficiency in human atherosclerosis and aortic aneurysms. J Clin Invest 1999, 104:1191-1197

21. Sukhova G, Shi G-P, Libby P: Role of elastolytic cathepsins in vascular remodeling. Edited by Matsuzawa YKT, Nagai R, Teramoto $T$. Kyoto, Japan, Elsevier Science BV, Amsterdam, 2004, pp. 498-501

22. Abdul-Hussien H, Soekhoe RG. Weber E, von der Thusen JH, Kleemann R, Mulder A, van Bockel JH, Hanemaaijer R, Lindeman JH: Collagen degradation in the abdominal aneurysm: a conspiracy of matrix metalloproteinase and cysteine collagenases. Am J Pathol 2007, 170:809-817

23. Lindholt JS, Erlandsen EJ, Henneberg EW: Cystatin C deficiency is associated with the progression of small abdominal aortic aneurysms. Br J Surg 2001, 88:1472-1475

24. Sukhova GS, Shi GP: Do cathepsins play a role in AAA pathogenesis? New York, Blackwell Publishing, 2006, pp. 161-169

25. Daugherty A, Manning MW, Cassis LA: Angiotensin II promotes atherosclerotic lesions and aneurysms in apolipoprotein E-deficient mice. J Clin Invest 2000, 105:1605-1612

26. Huh CG, Hakansson K, Nathanson CM, Thorgeirsson UP, Jonsson N, Grubb A, Abrahamson M, Karlsson S: Decreased metastatic spread in mice homozygous for a null allele of the cystatin $\mathrm{C}$ protease inhibitor gene. Mol Pathol 1999, 52:332-340

27. Daugherty A, Manning MW, Cassis LA: Antagonism of AT2 receptors augments angiotensin II-induced abdominal aortic aneurysms and atherosclerosis. Br J Pharmacol 2001, 134:865-870

28. Saraff K, Babamusta F, Cassis LA, Daugherty A: Aortic dissection precedes formation of aneurysms and atherosclerosis in angiotensin II-infused, apolipoprotein E-deficient mice. Arterioscler Thromb Vasc Biol 2003, 23:1621-1626

29. Wang B, Sun J, Kitamoto S, Yang M, Grubb A, Chapman HA, Kalluri R,
Shi GP: Cathepsin S controls angiogenesis and tumor growth via matrixderived angiogenic factors. J Biol Chem 2006, 281:6020-6029

30. Sukhova GK, Zhang $\mathrm{Y}$, Pan JH, Wada $\mathrm{Y}$, Yamamoto $T$, Naito $M$, Kodama T, Tsimikas S, Witztum JL, Lu ML, Sakara Y, Chin MT, Libby $P$, Shi GP: Deficiency of cathepsin S reduces atherosclerosis in LDL receptor-deficient mice. J Clin Invest 2003, 111:897-906

31. Sukhova GK, Wang B, Libby P, Pan JH, Zhang Y, Grubb A, Fang K, Chapman HA, Shi GP: Cystatin C deficiency increases elastic lamina degradation and aortic dilatation in apolipoprotein E-null mice. Circ Res 2005, 96:368-375

32. Lindholt JS, Heegaard NH, Vammen S, Fasting H, Henneberg EW Heickendorff L: Smoking, but not lipids, lipoprotein(a) and antibodies against oxidised LDL, is correlated to the expansion of abdominal aortic aneurysms. Eur J Vasc Endovasc Surg 2001, 21:51-56

33. Xiong W, Zhao Y, Prall A, Greiner TC, Baxter BT: Key roles of CD4+ $T$ cells and IFN-gamma in the development of abdominal aortic aneurysms in a murine model. J Immunol 2004, 172:2607-2612

34. Eliason JL, Hannawa KK, Ailawadi G, Sinha I, Ford JW, Deogracias MP. Roelofs KJ, Woodrum DT, Ennis TL, Henke PK, Stanley JC, Thompson RW, Upchurch GR Jr: Neutrophil depletion inhibits experimental abdominal aortic aneurysm formation. Circulation 2005, 112:232-240

35. Hall A, Ekiel I, Mason RW, Kasprzykowski F, Grubb A, Abrahamson M: Structural basis for different inhibitory specificities of human cystatins C and D. Biochemistry 1998, 37:4071-4079

36. Miwa K, Nakashima H, Aoki M, Miyake T, Kawasaki T, Iwai M, Oishi M, Kataoka K, Ongi S, Ogihara T, Kaneda Y, Morishita R: Inhibition of ets, an essential transcription factor for angiogenesis, to prevent the development of abdominal aortic aneurysm in a rat model. Gene Ther 2005, 12:1109-1118

37. Shi GP, Sukhova GK, Kuzuya M, Ye Q, Du J, Zhang Y, Pan JH, Lu ML, Cheng XW, Iguchi A, Perrey S, Lee AM, Chapman HA, Libby P: Deficiency of the cysteine protease cathepsin $S$ impairs microvessel growth. Circ Res 2003, 92:493-500

38. Li H, Cybulsky MI, Gimbrone MA Jr, Libby P: Inducible expression of vascular cell adhesion molecule- 1 by vascular smooth muscle cells in vitro and within rabbit atheroma. Am J Pathol 1993, 143:1551-1559

39. Pagano MB, Bartoli MA, Ennis TL, Mao D, Simmons PM, Thompson RW, Pham CT: Critical role of dipeptidyl peptidase I in neutrophil recruitment during the development of experimental abdominal aortic aneurysms. Proc Natl Acad Sci USA 2007, 104:2855-2860

40. Hoshina K, Koyama H, Miyata T, Shigematsu H, Takato T, Dalman RL, Nagawa $\mathrm{H}$ : Aortic wall cell proliferation via basic fibroblast growth factor gene transfer limits progression of experimental abdominal aortic aneurysm. J Vasc Surg 2004, 40:512-518

41. Ishisaka R, Utsumi T, Kanno T, Arita K, Katunuma N, Akiyama J, Utsumi K: Participation of a cathepsin L-type protease in the activation of caspase-3. Cell Struct Funct 1999, 24:465-470

42. Chwieralski CE, Welte T, Buhling F: Cathepsin-regulated apoptosis. Apoptosis 2006, 11:143-149

43. Stoka V, Turk B, Turk V: Lysosomal cysteine proteases: structural features and their role in apoptosis. IUBMB Life 2005, 57:347-353

44. Paik DC, Fu C, Bhattacharya J, Tilson MD: Ongoing angiogenesis in blood vessels of the abdominal aortic aneurysm. Exp Mol Med 2004, 36:524-533

45. Obermajer N, Doljak B, Kos J: Cysteine cathepsins: regulators of antitumour immune response. Expert Opin Biol Ther 2006, 6:1295-1309

46. Obermajer N, Repnik U, Jevnikar Z, Turk B, Kreft M, Kos J: Cysteine protease cathepsin $X$ modulates immune response via activation of beta2 integrins. Immunology 2008, 124:76-88

47. Kitamoto S, Sukhova GK, Sun J, Yang M, Libby P, Love V, Duramad $P$, Sun C, Zhang Y, Yang X, Peters C, Shi GP: Cathepsin L deficiency reduces diet-induced atherosclerosis in low-density lipoprotein receptor-knockout mice. Circulation 2007, 115:2065-2075

48. Tan SM, Hyland RH, Al-Shamkhani A, Douglass WA, Shaw JM, Law SK: Effect of integrin beta 2 subunit truncations on LFA-1 (CD11a) CD18) and Mac-1 (CD11b/CD18) assembly, surface expression, and function. J Immunol 2000, 165:2574-2581

49. van Noesel C, Miedema F, Brouwer M, de Rie MA, Aarden LA, van Lier RA: Regulatory properties of LFA-1 alpha and beta chains in human T-lymphocyte activation. Nature 1988, 333:850-852

50. Overall CM, Tam EM, Kappelhoff R, Connor A, Ewart T, Morrison CJ, Puente X, Lopez-Otin C, Seth A: Protease degradomics: mass spectrometry discovery of protease substrates and the CLIP-CHIP, a dedicated DNA microarray of all human proteases and inhibitors. Biol Chem 2004, 385:493-504 